
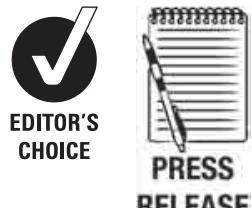

RELEASE

${ }^{1}$ Newcastle Neonatal Service, Royal Victoria Infirmary, Ward 35 , Royal Victoria Infirmary, Newcastle upon Tyne, UK ${ }^{2}$ Regional Maternity Survey Office, Newcastle upon Tyne, UK

\section{Correspondence to}

Dr Nicholas D Embleton, Newcastle Neonatal Service, Royal Victoria Infirmary, Newcastle upon Tyne NE1 4LP, UK;

n.d.embleton@newcastle. ac.uk

Accepted 15 February 2010

\title{
Survival in infants live born at less than 24 weeks' gestation: the hidden morbidity of non-survivors
}

\author{
Ravi Swamy, ${ }^{1}$ Sitikant Mohapatra, ${ }^{1}$ Mary Bythell, ${ }^{2}$ Nicholas D Embleton ${ }^{1}$
}

\section{ABSTRACT \\ Background Although survival rates for infants of} less than 26 weeks' gestation have increased, rates for those born at less than 24 weeks do not appear to have changed. While there are good data on overall survival, it is unclear how many infants are offered active resuscitation but do not survive. The study objectives were to describe the numbers receiving active treatment and the length of survival in infants live born at 22 or 23 weeks' gestation but who did not survive, and any changes over the last 15 years.

Methods The authors used a well-validated population-based database to identify deaths among live born infants born at 22 or 23 weeks' completed gestation between 1993 and 2007 from a single region in the north of England. The study period was divided into three 5-year cohorts. Survivors were identified from regional databases and individual case notes reviewed.

Results During the study period, there were 480662 total live births, of which 229 were live born at 22-23 weeks' gestation (birth prevalence of $0.05 \%$ ). Of the 210 infants who did not survive, 71 (34\%) survived for longer than $6 \mathrm{~h}$. The median survival of those who died but had received active resuscitation and were still alive at $6 \mathrm{~h}$ of age was $11 \mathrm{~h}$ in 1993-1997 ( $\mathrm{n}=17), 20 \mathrm{~h}$ in 1998-2002 $(n=28)$ and 3.7 days $(n=26)$ in 2003-2007.

Conclusion Over the last 15 years, increasing numbers of babies $<24$ weeks received active resuscitation. Overall survival has not changed, but non-survivors endured significantly longer durations of intensive care.

\section{INTRODUCTION}

Recent data have shown that the overall survival for extremely preterm infants born $\geq 24$ weeks has increased. ${ }^{12}$ The gestational age at which active care should be provided remains extremely contentious, ${ }^{3}$ and guidelines have been produced in the UK in order to aid management decisions. ${ }^{4}$ Despite this, practice is likely to vary. In addition, although there are good population-based data on overall survival, it is not clear how many babies at the margins of viability ( $<24$ weeks) are offered active treatment and whether this has changed in association with the increasing survival seen in all infants $<26$ weeks over the last decade. ${ }^{12}$

Our objectives were to document survival in live born infants at 22 or 23 weeks' gestation, to ascertain how many received active treatment and to document the length of survival for those who died. We also aimed to describe interventional procedures in the most recent time period (as a very crude proxy for suffering) both in those who survived and in those who died.

\section{METHODS}

We used the Perinatal Mortality Survey (PMS), a well-validated population-based database coordinated by the Regional Maternity Survey Office (RMSO), ${ }^{6}$ to identify deaths prior to discharge among live born infants at 22 or 23 weeks of completed gestation between January 1993 and December 2007. We cross-checked this with the regional database supplied from the four tertiary neonatal units in this region where all possible regional survivors will have received care. We did not include babies born to mothers who were resident outside the Northern region but delivered in the region.

We reviewed data held in the PMS and utilised existing unit databases (that were further reviewed by local clinicians if necessary) to obtain the following information: gestation, antenatal risk factors, mode of delivery, initial condition at birth, resuscitation details, major neonatal morbidities and management. Because we were not sure how accurately we would be able to ascertain retrospectively whether active resuscitation was unsuccessful (but had been the intention), we decided to identify the subgroup of infants who were still alive at 6 h of age. These are all likely to have been alive as a consequence of an active decision to provide resuscitation and ongoing care, and might enable a more robust comparison between different time periods. We acknowledge that there will have been babies who were actively resuscitated but failed to survive beyond $6 \mathrm{~h}$.

In order to describe trends over time, we arbitrarily divided the period into three 5-year time periods, 1993-1997, 1998-2002 and 2003-2007, and plotted Kaplan-Meier survival curves (using SPSS 16.0, SPSS, Chicago, Illinois) for infants who were still alive at $6 \mathrm{~h}$ of age. A detailed analysis of the last epoch was used to describe morbidity and interventions undergone by both survivors and non-survivors.

We only retrieved anonymised data for survivors from unit databases not receiving care at our unit, and used data already held by the PMS on non-survivors and therefore did not apply for formal ethics committee approval. The RMSO has a process for project review, through which we were allowed access to the relevant data. The PMS has approval from the Patient Information Advisory Group to collect patient data under Section 60 of the Health and Social Care Act (2001). This piece of research falls within the existing ethical approval framework for work carried out at the RMSO. 


\section{RESULTS}

During the study period, there were 480662 total live births, of whom 229 were live born at 22 or 23 weeks' gestation. This amounts to a birth prevalence of $0.05 \%$. In the first epoch, there were 85 live births (31 born at 22 weeks' gestation), 66 in the second (16 born at 22 weeks) and 78 in the third (26 born at 22 weeks) (see table 1). Only one infant was delivered by caesarean section in this entire cohort.

Of the 210/229 (92\%) who did not survive, there were 21 pairs of twins and one set of triplets. Seventy-one (34\%) of these non-surviving infants lived at least $6 \mathrm{~h}$ and were admitted to a tertiary neonatal unit, 23/71 were born in a non-tertiary neonatal unit hospital, and 14/71 were born at 22 weeks. The median survival for these infants increased during the study period: $11 \mathrm{~h}$ in 1993-1997 ( $\mathrm{n}=17), 20 \mathrm{~h}$ in 1998-2002 $(\mathrm{n}=28)$ and 3.7 days $(\mathrm{n}=26)$ in 2003-2007. The survival curves for infants alive at $6 \mathrm{~h}$ are shown in figure 1 . The age in days has been truncated to 225 days for the ease of presentation.

In the final epoch, there was clear documentation that $46 / 77(60 \%)$ infants had been actively resuscitated, of whom 43 infants survived for at least $6 \mathrm{~h}$. Surgical procedures were performed in 5/43 (12\%) non-survivors: laparotomy $(n=3)$, patent ductus arteriosus (PDA) ligation $(n=1)$ and laser treatment for retinopathy of prematurity $(R O P)(n=1)$. There were 19/229 (8\%) survivors during the entire period: six (19931997), six (1998-2002) and seven (2003-2007), of whom only one was born at 22 weeks. Of the seven survivors in the last

Table 1 Total deliveries and survival

\begin{tabular}{lcccr}
\hline & 1993-1997 & 1998-2002 & 2003-2007 & \multicolumn{1}{c}{ Total } \\
\hline $\begin{array}{l}\text { Spontaneous abortions } \\
\text { (22-23 weeks) }\end{array}$ & 198 & 154 & 143 & 495 \\
Total deliveries & 85 & 66 & 78 & 229 \\
$\begin{array}{l}\text { (22-23 weeks) } \\
\text { Total live born deliveries }\end{array}$ & 172022 & 150659 & 157981 & 480662 \\
$\begin{array}{l}\text { Infants (22-23 weeks) } \\
\text { who survived }>6 \text { h }\end{array}$ & 23 & 34 & 33 & 90 \\
$\begin{array}{l}\text { Infants (22-23 weeks) who } \\
\text { survived }>6 \text { h and then died }\end{array}$ & 17 & 28 & 26 & 71 \\
\hline
\end{tabular}

\section{Kaplan Meier survival for infants alive at $6 \mathrm{~h}$ of age}

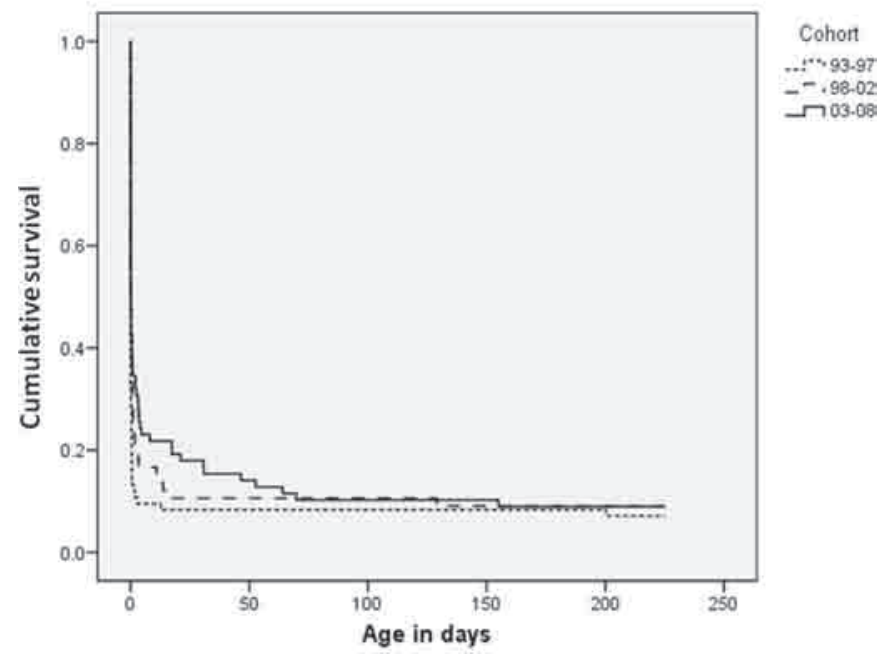

Figure 1 Kaplan-Meier Survival Plot for babies born $<24$ weeks' gestation and alive at $6 \mathrm{~h}$ of age. Age is in days truncated to 225 days for ease of presentation. epoch, four babies required surgical procedures: laparotomy for necrotising enterocolitis (NEC) $(n=1)$, PDA ligation $(n=3)$ and laser treatment for $\mathrm{ROP}(\mathrm{n}=3)$.

\section{DISCUSSION}

These data are consistent with other recently published series showing that survival at $<24$ weeks does not appear to have changed significantly, in contrast to increases for those born at 24 or more weeks. ${ }^{12}$ This has been paralleled by an increase in the number of infants $<24$ weeks being offered active resuscitation, and an increased length of time non-survivors received active, but ultimately unsuccessful, intensive care.

We used a well-validated database to identify cases from a large geographical region but were unable to clearly identify factors that might predict non-survivors at an early stage although the numbers in our series are small. There was no guideline or formal agreement throughout the region as to whether active resuscitation should be offered (or not), but there did not appear to be systematic differences between units. Our reliance on review of case records to determine whether active resuscitation was planned may mean that we underascertained active attempts, but using our criteria of being alive at $6 \mathrm{~h}$, we enabled a more robust comparison over time.

Our local (anecdotal) experience is that most parents request an active approach immediately after delivery at 23 weeks' gestation, despite counselling from experienced clinicians using nationally consistent survival data. While parents and clinicians may recognise that survival rates $<24$ weeks are low, these current data emphasise the increasing amounts of intensive care endured by those who do not survive infancy and might be useful when parents are counselled.

Future research directed at identifying robust prognostic factors for non-survival in the first days and weeks may limit prolonged unsuccessful intensive care but requires a sufficiently large population base given the low prevalence. Some will feel that the prolonged periods of intensive, but unsuccessful, care demonstrated in this report are either futile or inappropriate. An improved understanding of societal and parental attitudes and perceptions towards either the withholding or the withdrawing of active treatment at the margins of viability is needed.

Acknowledgements The authors gratefully acknowledge all the clinicians and coordinators who have contributed data to the Perinatal Mortality Survey.

Funding Regional Maternity Survey Office is funded by the primary healthcare trusts of the North East of England.

Competing interests None.

Provenance and peer review Not commissioned; externally peer reviewed.

\section{REFERENCES}

1. EPICure 2 Perinatal Group. Survival and early morbidity of extremely preterm babies in England: changes since1995 Arch Dis Child 2008;93: (Supp 1):A33-4

2. Field DJ, Dorling JS, Manktelow BN, et al. Survival of extremely premature babies in a geographically defined population: prospective cohort study of 1994-9 compared with 2000-5. BMJ 2008;336:1221-3.

3. Nuffield Council on Bioethics. Critical care decisions in fetal and neonatal medicine: ethical issues. 2006.

4. Wilkinson AR, Ahluwalia J, Cole A, et al. Management of babies born extremely preterm at less than 26 weeks of gestation: a framework for clinical practice at the time of birth. Arch Dis Child Fetal Neonatal Ed 2009:94:F2-5.

5. Northern Regional Health Authority Coordinating Group. Perinatal mortality: a continuing collaborative regional survey. BMJ 1984;288:1717-20.

6. Northern Regional Health Authority Coordinating Group. Northern Regional Maternity Survey Office. Annual report 2000. Newcastle upon Tyne, 2002. 